\title{
Analyse de risques pour la maintenance des structures portuaires : exemple de quais gabions soumis à de la corrosion.
}

\author{
Jérôme Boéro ${ }^{*+}$, Bruno Capra*, Franck Schoefs ${ }^{+}$, Olivier Bernard*, \\ Marc Lasne* \\ * OXAND S.A, 36 bis, avenue Franklin Roosevelt, 77210 Avon \\ ${ }^{+}$Institut de Recherche en Génie Civil et Mécanique (GeM), UMR 6183, \\ Faculté Sciences et Techniques, 2 rue de la Houssinière, 44000 Nantes
}

Résumé : L'article qui suit présente la mise en œuvre d'une méthodologie d'analyse de risques afin d'optimiser la gestion de parcs d'ouvrages de génie civil à fort enjeu. Pour illustrer ce savoir-faire, une analyse de risques d'un quai gabions vis à vis de la corrosion en milieu marin est exposée. La démarche consiste à identifier et hiérarchiser les modes de défaillances en fonction de leur criticité (Analyse de Risques Qualitative). Une Analyse de Risques Quantitative détermine alors, de manière plus fine, la probabilité de défaillances des modes les plus critiques. L'article se conclut sur la nécessité de mettre en place des plans de maintenance de base et plus élaborés, conditionnels aux mesures et calculs effectués.

Abstract : This article presents a methodology of risk analysis developed to optimize the management of civil engineering structures of strong stake. To illustrate this know-how, a risk analysis of a cellular cofferdam type quay wall taking into account corrosion in marine environment is presented. The methodology consists to identify and classify the failures modes according to their criticity (Qualitative Risk Analysis). Then, a Quantitative Risk Analysis determines the probability of failure of the most critical modes. The article ends by the need for setting up basic and more elaborate maintenance plans related to measurements and calculations carried out.

Mots clés : corrosion électrolytique, corrosion biologique, quais, palplanches, analyse de risques, maintenance.

Keywords : electrolytic corrosion, biological corrosion, quays, sheet piling, risk analysis, maintenance. 


\section{Introduction}

Les gestionnaires de structures portuaires sont amenés à planifier des opérations de maintenance (surveillance et entretien) sur un patrimoine hétérogène et comprenant un nombre important d'ouvrages aux fonctions variées. La maintenance curative étant en général très coûteuse en coûts directs comme en conséquences indirectes (arrêts d'exploitation, ...), le recours à une méthodologie permettant d'anticiper les interventions et de hiérarchiser les priorités est de première importance. Les approches de type risque [VIL88] sont en plein essor car elles permettent de répondre à ce besoin méthodologique et intègrent les évolutions des phénomènes dans le temps (corrosion, trafic portuaire, ...), ce qui est impératif dès qu'il s'agit d'élaborer des plans pluriannuels de maintenance.

Cette approche est illustrée au travers d'un exemple de planification de la maintenance d'un quai gabions soumis à de la corrosion.

\section{Les analyses de risques pour la planification de la maintenance des} ouvrages portuaires

\subsection{Démarche de l'analyse de risques}

L'analyse de risques menée sur le quai gabions comporte trois étapes :

- Etape 1 : une Analyse de Risques Qualitative (ARQL) afin d'inventorier de manière exhaustive les Modes de Défaillance (MdD) et de les hiérarchiser à l'aide d'un indicateur de risques, la Criticité.

- Etape 2 : une Analyse de Risques Quantitative (ARQN) pour évaluer les probabilités associées aux principaux modes de défaillance.

- Etape 3 : un cadrage de la maintenance.

\subsection{Application de l'analyse de risques à un quai gabions}

L'ouvrage étudié est un quai constitué de gabions circulaires juxtaposés, reliés par des festons formant un arc (cf. Figure 1).

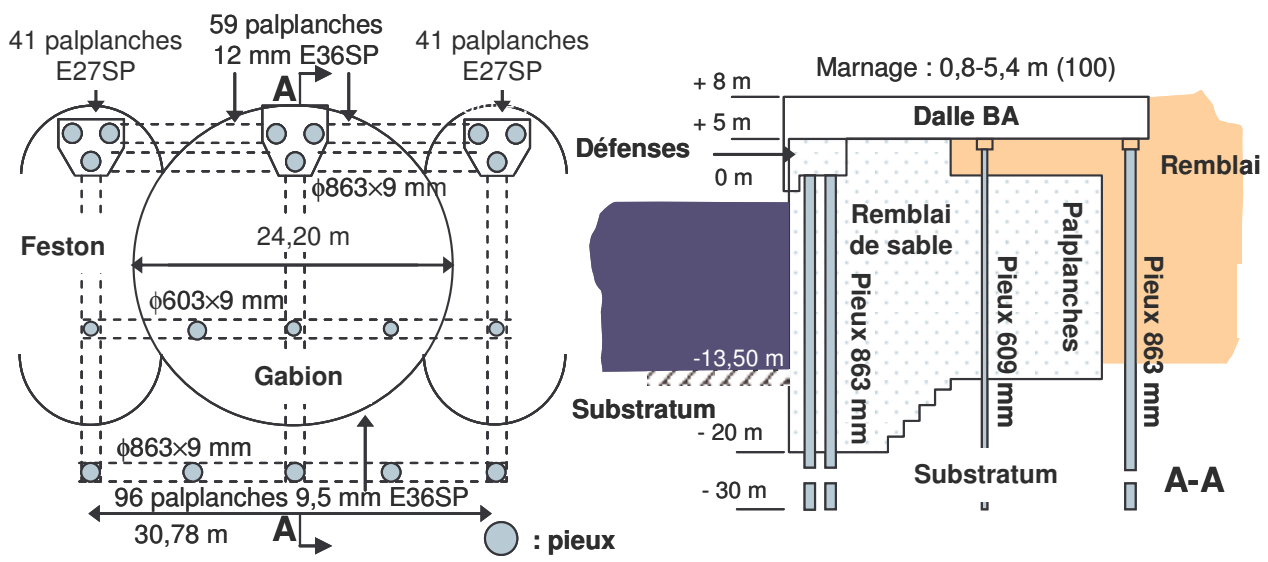

Figure 1 : Coupe-type et vue en plan du segment représentatif du quai 
Le système «quai gabions » est composé d'une fondation en gabions et en festons de palplanches, un remblaiement de sable, des pieux creux en acier battus dans le sable jusqu'au niveau du substratum, une dalle nervurée en béton armé et des défenses d'accostage.

\section{L'Analyse de Risques Qualitative (ARQL)}

\subsection{L'analyse fonctionnelle (AF)}

L'analyse fonctionnelle décrit le fonctionnement du système étudié afin de faciliter sa compréhension. L'analyse fonctionnelle externe (AFE) permet de faire un inventaire exhaustif des milieux extérieurs en contact avec le système (cf. Figure 2) et de déterminer les réponses du système face aux sollicitations imposées par son environnement (fonctions de contrainte externes).

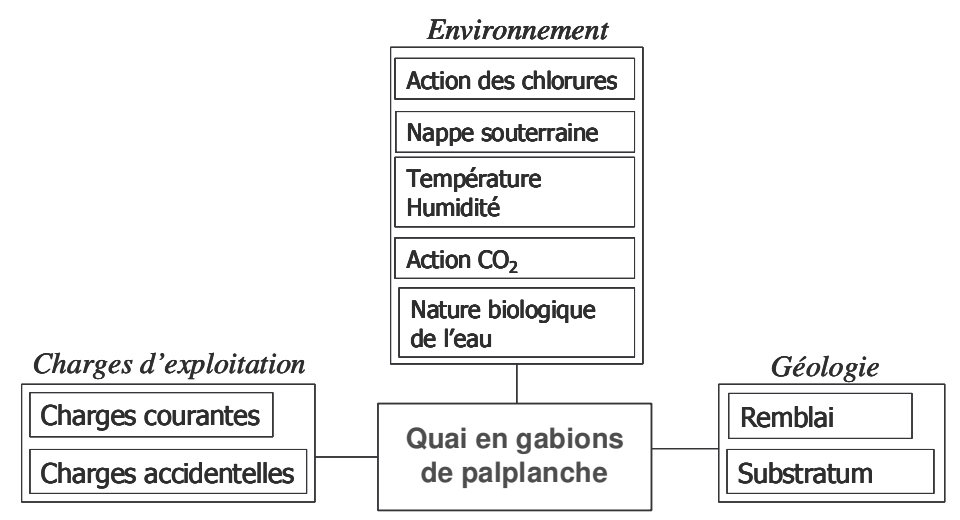

Figure 2 : Diagramme fonctionnel du système global « quai en gabions de palplanches » dans son environnement

L'Analyse Fonctionnelle Interne (AFI) permet de réaliser un inventaire des composants du système (cf. $§ 2.2$ ) et de définir les interactions possibles entre eux (fonctions de contraintes internes). Dans la suite de l'article, les fonctions de contraintes externes et internes sont regroupées sous le terme de «fonctions de contraintes ». A titre d'exemple, la dalle nervurée en béton a pour fonction de contraintes, de résister aux charges d'exploitation et accidentelles, et de les transmettre aux pieux.

Etant donné le caractère répétitif de la structure du quai gabions, l'analyse de risques se concentre sur un segment représentatif, à savoir un gabion et deux festons (cf. Figure 1).

\subsection{Inventaire et hiérarchisation des modes de défaillance}

A l'aide de la méthode de l'AMDEC (Analyse des Modes de Défaillance, de leurs Effets et de leurs Criticités), le travail consiste maintenant, pour chaque fonction de contraintes, à déterminer le mode, les causes, les effets, les symptômes et les moyens de détection de la défaillance. Un mode de défaillance étant une fonction de contraintes qui n'est plus assurée. 
Une fois les modes de défaillance identifiés, ils sont hiérarchisés grâce à leur criticité. L'obtention du degré de criticité (ou niveau de risque) de chaque mode de défaillance se fait par la combinaison des 4 notes suivantes :

- la fréquence F d'apparition (probabilité d'occurrence) d'un mode de défaillance ;

- la gravité G (coût) liée aux conséquences d'un mode de défaillance réalisé ;

- les moyens de détection $\mathrm{D}$ des signes annonciateurs d'une défaillance ;

- l'évolution potentielle dans le temps E.

Pour le quai gabions étudié, 16 modes de défaillance (MdD) ont été identifiés. Les modes de défaillance les plus critiques sont listés ci-dessous (cf. Tableau 1).

Tableau 1 : Modes de défaillance les plus critiques pour le quai gabions étudié

\begin{tabular}{|l|l|l|}
\hline \multicolumn{1}{|c|}{ Composant } & \multicolumn{1}{|c|}{ Fonction } & \multicolumn{1}{c|}{ Modes de défaillance } \\
\hline \multirow{2}{*}{ Pieux en acier } & $\begin{array}{l}\text { Résister aux charges d'exploitation } \\
\text { transmises par la dalle }\end{array}$ & $\begin{array}{l}\text { Le pieu n'est plus en mesure de transmettre l'effort } \\
\text { provenant de la dalle nervurée en béton armé }\end{array}$ \\
\hline \multirow{2}{*}{$\begin{array}{l}\text { Palplanches du gabion } \\
\text { et des festons }\end{array}$} & Contenir le sable de remblaiement & $\begin{array}{l}\text { Le sable fuit au travers des palplanches par des } \\
\text { percements et/ou au droit de joints entre palplanches }\end{array}$ \\
\cline { 2 - 3 } Remblaiement de sable & $\begin{array}{l}\text { Isoler le sable de remblaiement de l'eau de } \\
\text { mer }\end{array}$ & $\begin{array}{l}\text { L'eau de mer pénètre dans le sable contenu dans les } \\
\text { gabions et festons }\end{array}$ \\
\cline { 2 - 3 } & $\begin{array}{l}\text { Assurer la stabilité du gabion et/ou des } \\
\text { poussée du remblai situé derrière }\end{array}$ & $\begin{array}{l}\text { La poussée des remblais derrière les palplanches } \\
\text { entraîne une perte de stabilité du gabion et/ou des } \\
\text { festons }\end{array}$ \\
\cline { 2 - 3 } & $\begin{array}{l}\text { Contribuer à la capacité portante des pieux } \\
\text { par frottement latéral }\end{array}$ & $\begin{array}{l}\text { Le sable ne contribue plus à la capacité portante des } \\
\text { pieux par frottement latéral }\end{array}$ \\
\hline
\end{tabular}

L'ARQL menée au niveau du système a mis en évidence que :

- les mécanismes de corrosion (percements et/ou dégrafages des palplanches) sont à l'origine des modes de défaillance les plus critiques pour le quai étudié ;

- les modes de défaillance des pieux en acier et du remblaiement de sable sont principalement conditionnés par les modes de défaillance des palplanches. Notamment, par celui associé à la fonction « contenir le sable de remblaiement». Le quai gabions étudié comporte un nombre important de pieux positionné à proximité du front d'accostage (zone qui, en cas de fuite de sable au travers des gabions et/ou festons de palplanches, devrait être vidangée en priorité). En terme de risque, les pertes d'exploitation les coûts de maintenance (défaillance des rails et des portiques) pilotent l'étude car elles priment sur les questions de stockage auxquelles sont associées la planéité de la plateforme. Cette dernière est elle-même influencée par la perte de matériau, donc de poids, et par la perte de stabilité de l'enceinte gabion sous la poussée des terres. Sous l'hypothèse que ces deux phénomènes sont indépendants, la perte d'exploitation du quai, liée à la perte de capacité portante des pieux (frottement latéral), est privilégiée.

A ce stade de l'analyse, une approche quantitative est nécessaire pour :

- évaluer les cinétiques possibles de corrosion au niveau des palplanches ;

- quantifier la perte de capacité portante des pieux en frottement latéral. 


\section{Analyse de Risques Quantitative (ARQN) des palplanches et des pieux}

\subsection{Etude quantitative du percement des palplanches corrodées}

\subsubsection{Impact de la corrosion sur les palplanches}

Les mécanismes de corrosion électrolytique et leurs cinétiques diffèrent suivant la hauteur du quai. En effet, depuis plusieurs années, il a été montré que les structures immergées en acier au carbone, subissent une corrosion électrolytique maximale localisée dans les plus basses eaux de la zone de marnage et la zone d'éclaboussure [MEM00], [BEN04].

La corrosion biologique, autre mécanisme de corrosion rencontré en milieu marin, se produit principalement dans les zones immergées et au niveau de l'encastrement des palplanches dans le sol. La zone de marnage semble être épargnée par la biocorrosion, à cause des cycles de séchage/mouillage qui empêchent la fixation du biofilm à la surface de l'acier. Ce mécanisme de corrosion, bien qu'il soit localisé (piqûres), est la cause de nombreux désordres sur des structures portuaires métalliques, car il se développe rapidement (cinétique de corrosion jusqu'à 10 mm/an).

\subsubsection{Modélisation probabiliste du percement des palplanches par biocorrosion}

En fonction des mécanismes de corrosion cités précédemment, différentes zones de l'ouvrage sont identifiées. Pour chacune d'entre elles, des cinétiques de corrosion sont évaluées par avis d'expert afin de servir de données d'entrée à la modélisation des mécanismes de corrosion des palplanches. Pour pallier le manque de données d'inspection, les modèles de cinétiques tiennent compte d'incertitudes.

Une évaluation jusqu'en 2020 de fuseaux de perte d'épaisseur de palplanches par corrosion électrolytique et par corrosion biologique est effectuée pour chaque zone. Deux valeurs moyennes sont envisagées pour chaque type de corrosion: une borne basse et une haute. Les bornes obtenues par ces modélisations montrent que l'impact de la corrosion biologique domine celui de la corrosion électrolytique $(0,5$ à $4,5 \mathrm{~mm}$ pour une épaisseur initiale de $12 \mathrm{~mm})$. On cherche donc par la suite à préciser cette approche par une modélisation probabiliste. On présente ci-dessous les résultats du phénomène de corrosion biologique, identifié comme majeur. La probabilité de percement des palplanches par corrosion biologique est calculée à l'aide du logiciel SIMEO ${ }^{\mathrm{TM}}$ Consulting [SIM03]. Deux hypothèses de valeurs de vitesse moyenne sont choisies car le retour d'expérience montre que la plage de vitesses de corrosion biologique est étendue. La vitesse de corrosion biologique est supposée constante (hypothèse conservative) et probabilisée par une vitesse moyenne basse de 0,5 $\pm 0,5 \mathrm{~mm} / \mathrm{an}$ et une vitesse moyenne haute de 1,0 $\pm 0,5 \mathrm{~mm} / \mathrm{an}$. Deux lois de distribution triangulaires sont utilisées pour décrire les incertitudes sur les vitesses de corrosion biologique. Sachant physiquement que les probabilités d'occurrence des vitesses sont plus faibles sur les bords du support (valeurs extrêmes), borné par ailleurs, ce type de 
loi de distribution remplit ces conditions. D'autres lois peuvent être envisagées sur cette base avec une étude de sensibilité à réaliser si l'étude de risque montre des criticités trop semblables. Il faut toutefois voir cette loi comme une loi support destinée à être actualisée notamment par approche bayésienne [LED05].

La probabilité de percement des palplanches est calculée par la méthode de simulation de Monte-Carlo [LEM05]. La figure ci-dessous présente une évaluation du fuseau dans lequel se situent les probabilités de percement des palplanches par corrosion biologique obtenues par simulation (cf. Figure 3).

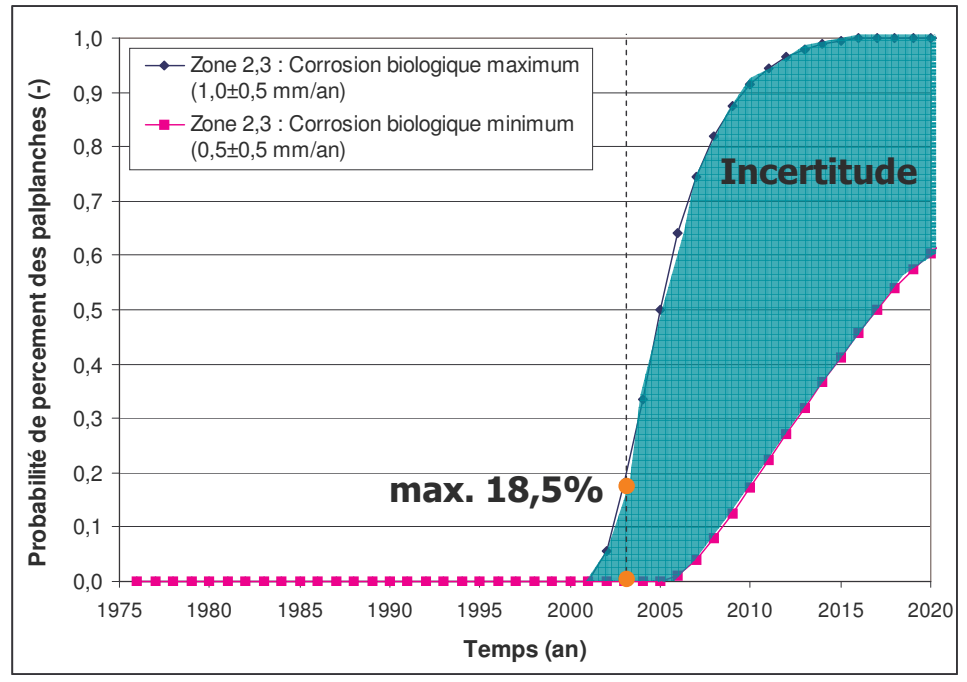

Figure 3 : Probabilités de percement des palplanches par corrosion biologique

On constate que l'hypothèse de vitesse moyenne haute implique une probabilité de défaillance de $18,5 \%$ en 2003 et que l'écart entre les deux hypothèses peut atteindre $70 \%$ vers 2010 . Les résultats obtenus montrent que la stratégie de maintenance du quai repose sur la présence ou non de bactéries au droit des palplanches.

\subsection{Etude quantitative de la perte de capacité portante des pieux en frottement latéral}

Ces questions sont extrêmement complexes et ne sont traités ici que les enjeux et ordres de grandeurs obtenus. La perte de portance des pieux en frottement latéral est influencée par de multiples facteurs :

- la fuite du remblaiement de sable à partir des gabions et des festons ;

- la corrosion biologique (rupture d'un ou plusieurs pieux par percements localisés (état limite d'instabilité interne)) et électrolytique (perte d'adhérence entre l'acier et le sable et/ou entre l'acier et le substratum par formation de couches d'oxydes (état limite d'instabilité externe)) des pieux ;

- la pénétration de l'eau de mer dans le remblaiement de sable qui diminue le frottement latéral des pieux en réduisant la contrainte effective dans le sable et exerce cycliquement (marnage) sur les grains de sable une force hydrodynamique qui entraîne la vidange de gabions et/ou festons [SCH03]. 
Pour le quai étudié, la complexité réside donc dans la définition de l'impact de chacun des facteurs cités ci-dessus sur la perte de portance des pieux en frottement latéral.

Pour cela, une étude géotechnique préliminaire, selon le fascicule 62 [JO93], [ROS00], est entreprise. La résistance axiale des pieux vis à vis des charges verticales se compose du frottement latéral du remblaiement de sable et du substratum, ainsi que de la pression de pointe. Dans le cas étudié, la résistance de pointe $(100 \mathrm{kPa})$ est négligeable par rapport à la résistance latérale.

A défauts de données d'inspection, plusieurs scénarios extrêmes sont élaborés à partir des facteurs de corrosion décrits dans le §4.2.1. Les résultats montrent que la perte de portance des pieux en frottement latéral, situés à proximité du front d'accostage, avoisine $27 \%$ et peut atteindre $100 \%$ en cas de corrosion dans le substratum. Par conséquent, la détection de chaque facteur conditionne l'élaboration du plan de maintenance du quai étudié.

Par la suite, à l'aide de données d'inspections et d'une meilleure connaissance de la propagation de certains phénomènes dans les milieux non-inspectables, des scénarios plus précis pourront être envisagés. La surface de pieux menacée par une perte de frottement latéral pourra alors être évaluée à l'aide d'une analyse probabiliste. A l'issu de ce travail, le plan de maintenance du quai gabions pourra alors être actualisé, et par conséquent optimisé.

\section{Cadrage de la Maintenance}

Dans ce qui suit, pour pallier le manque d'informations sur les mécanismes de corrosion et leurs cinétiques, des recommandations de maintenance (surveillance et entretien) sont présentées.

Les inspections ont pour objectif de :

- lever l'incertitude sur l'existence de la corrosion biologique à l'aide d'une inspection des palplanches par des plongeurs spécialisés ;

- mesurer l'avancement de la corrosion électrolytique à la surface des palplanches à l'aide de mesures résiduelles d'épaisseurs ;

- mesurer l'état de corrosion des pieux battus dans les gabions et festons.

Cette inspection est conditionnée par la présence de corrosion biologique sur les palplanches.

Outre le fait d'actualiser les hypothèses sur les modèles de cinétiques de corrosion, formulées par les experts, ces inspections pilotent les actions d'entretien à réaliser pour maintenir les conditions de sécurité et d'exploitation du quai. Sur la base des données disponibles, et à l'issu des résultats de l'analyse de risques du quai gabions, le plan d'entretien de base consiste à réactiver la protection cathodique existante ou à mettre en œuvre une nouvelle protection cathodique (action préventive). D'autres plans plus élaborés et conditionnels complètent ce dispositif en fonction des résultats des différentes inspections décrites précédemment. 


\section{Conclusion}

L'analyse de risques qualitative menée au niveau du quai gabions a mis en évidence que les mécanismes de corrosion électrolytique (chlorures) et biologique (bactéries) sont à l'origine des modes de défaillance les plus critiques pour le quai étudié.

L'analyse de risques quantitative a permis de constater que les risques associés à un percement de palplanches dépendent principalement des mécanismes de corrosion biologique. La conséquence ultime de cette défaillance réside dans la fuite partielle ou totale du sable contenu dans les gabions et/ou festons. Par ailleurs, les risques associés à la défaillance des pieux résultent de la fuite de sable et/ou de la corrosion des pieux, dont la cinétique dépend en grande partie de la pénétration de l'eau de mer dans le remblaiement de sable. Ces phénomènes ont pour conséquences de diminuer, voir même d'annuler totalement la capacité portante des pieux en frottement latéral, ce qui peut se traduire par une perte de stabilité du quai.

La complexité du problème montre l'intérêt d'une approche par les risques qui apporte des éléments d'aide à la décision et permet d'aboutir à des plans de maintenance priorisés.

\section{Bibliographie}

[BEN04], Bénaissa B., (2004), La corrosion des structures métalliques en mer: types et zones de dégradations, VIIIèmes Journées Nationales Génie Civil - Génie Côtier, Compiègne, 7-9 septembre 2004.

[JO93], JO 23/03/93, Décret 93-446: CCTG Fascicule 62 Titre V, Règles techniques de conception et de calcul des fondations des ouvrages de génie civil.

[LED05], Le Drogo J., Capra B., Wolff V., (2005), Applications des réseaux bayésiens au vieillissement des structures du génie civil, Fiabilité des matériaux et des structures, $4^{\text {ème }}$ conférence nationale « JNF'05 », Clermont-Ferrand, France.

[LEM05], Lemaire M., Chateauneuf A., Mitteau J-C., (2005), Fiabilité des structures : couplage mécano-fiabiliste statique, Hermès Science Publication, 249282.

[MEM00], Mémet J.B., (2000), La corrosion des structures métalliques portuaires : étude des mécanismes d'amorçage et de croissance des produits de corrosion, Thèse de doctorat, Université de La Rochelle.

[ROS00], Logiciel ROSA 2000, CETMEF, (2001), Recommandations pour le calcul aux états-limites des ouvrages en site aquatique.

[SCH03], Schoefs F., Rémond F., (2003), Etude de risque appliquée à un quai en gabions soumis à de la corrosion, Institut de Recherches en Génie Civil et Mécanique, Rapport interne $\mathrm{n}^{\circ} 03-11$-int-02, 89 pages.

[SIM03], Logiciel SIMEO ${ }^{\mathrm{TM}}$ Consulting 1.0, OXAND S.A., (2003), Avon, France.

[VIL88], Villemeur A., (1988), Sûreté de fonctionnement des systèmes industriels, Eyrolles. 